\title{
Ventilation Heterogeneity and Airway Hyperreactivity in Children with Well Controlled Asthma
}

Santiago José Assaf ${ }^{1}$, Charles Curtis Clem ${ }^{1}$, Lauren Bockstahler Jewett ${ }^{1}$, Leah Schornick ${ }^{1}$, Christina Jo Tiller ${ }^{1}$, Jeffrey A Kisling ${ }^{1}$, Stephanie D Davis ${ }^{1 *}$ and Robert $S$ Tepper ${ }^{1,2}$

${ }^{1}$ Department of Pediatrics, Section of Pediatric Pulmonology, Allergy and Sleep Medicine, Riley Children's Hospital, Indiana University, Indianapolis, Indiana, USA ${ }^{2}$ Herman B. Wells Center for Pediatric Research, Indianapolis, Indiana, USA

\section{Abstract}

Rationale: In asthma, airway inflammation, obstruction and reactivity may lead to ventilation heterogeneity; our understanding of this process is limited in asthmatic children.

Objectives and Methods: The study's objective was to measure ventilation heterogeneity, by the lung clearance index ( $\mathrm{LCl})$, in children with well controlled asthma and assess the association of $\mathrm{LCl}$ with airway reactivity through methacholine challenge tests. $\mathrm{LCl}$ and spirometry were measured in 24 children with asthma and 21 healthy controls between 4 and 10 years of age. Sixteen children with asthma and 11 healthy controls also performed methacholine challenge tests

Results: LCl was higher in children with asthma compared to healthy controls (7.58 vs. 6.79, $p=0.004)$; no differences in $\mathrm{FEV}_{1}$ or $\mathrm{FEF}_{25-75}$ were noted between groups. Ages were similar $(p=0.54)$; however, the slope of LCl versus age differed between groups $(p=0.001)$. The LCl slope increased with age in asthmatics. Further, higher LCI values were associated with decreasing $\mathrm{PC}_{20}$ (provocative concentration of methacholine to decrease baseline $\mathrm{FEV}$, by $20 \%$ ) values in children with asthma $(p=0.02)$, but not healthy controls $(p=0.16)$.

Conclusions: Ventilation heterogeneity is present from preschool age in children with well controlled asthma. The relationship between ventilation heterogeneity and airway reactivity suggests that normalizing ventilation heterogeneity may be an important therapeutic target for treating children with asthma.

Keywords: Ventilation inhomogeneity; Lung clearance index; Spirometry; Airway reactivity; Asthma

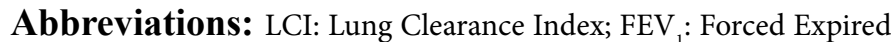
Volume in One Second; $\mathrm{FEF}_{25-75}$ : Forced Expired Flows between 25 and 75 Percent of Forced Vital Capacity

\section{Introduction}

Asthma is characterized by recurrent episodes of airway obstruction, hyperreactivity, and inflammation, which may contribute to airway remodeling and progressive flow limitation [1,2]. Autopsy and biopsy specimens have demonstrated increased airway inflammation and remodeling in the smaller, more peripheral airways [3]; however, obstruction in these airways may not be detected using spirometry. Further, asthma is characterized by inhomogeneity of airway disease that can produce ventilation heterogeneity within the lung that is not readily detectable with spirometry.

The multiple breath inert gas washout technique (MBW) can assess ventilation heterogeneity within the lung by calculating the lung clearance index (LCI) [4]. This technique quantifies the washout of an inert gas during regular tidal breathing, making it suitable for all ages, particularly young children who may not perform reproducible spirometry [5]. Studies have demonstrated that well-controlled adults with asthma may have normal spirometry, but an elevated LCI, reflecting greater ventilation heterogeneity [6,7]. Among adult asthmatics, greater ventilation heterogeneity is associated with increased airway reactivity [8]. Limited studies have been conducted in children with asthma evaluating ventilation heterogeneity using differing inert gases (sulfur hexafluoride, $\mathrm{SF}_{6}$; and nitrogen, $\mathrm{N}_{2}$ ) and methodology [9-12]. No studies have specifically evaluated the association of ventilation heterogeneity with airway hyper-responsiveness in children with asthma. We hypothesized that pre-school and school age children with well-controlled asthma have evidence of ventilation heterogeneity and that school age children with well-controlled asthma have increasing ventilation heterogeneity associated with greater airway reactivity.

\section{Materials and Methods}

\section{Subjects}

Asthmatic children: Subjects between 4 and 10 years of age diagnosed with asthma by a pediatric pulmonologist were enrolled from the pediatric pulmonary clinics at Riley Hospital for Children (Indiana University School of Medicine). Inclusion criteria included mild to moderate asthma according to the Global Initiative for Asthma (GINA) 2010 symptoms and treatment criteria [13], with spirometry values of forced expiratory volume in one second $\left(\mathrm{FEV}_{1}\right) \geq 80$ percent predicted. Subjects also had well controlled disease at the time of testing, defined by the absence of an acute exacerbation and treatment modifications for 4 weeks prior to testing. In addition, parents completed the Asthma Control Test and subjects with scores $<20$ (poor control) were excluded [14].

Healthy controls: Subjects between 4 and 10 years of age were recruited through advertisements in local publications in Indianapolis, Indiana (2013-2014). Inclusion criteria included being full term $(\geq 37$ weeks of gestation) and no history of allergic rhinitis, food allergy, atopic dermatitis, wheezing, pneumonia, or hospitalization for a respiratory illness. Exclusion criteria included any history of immunodeficiency,

*Corresponding author: Stephanie D. Davis, MD, Section of Pediatric Pulmonology, Allergy and Sleep Medicine, Riley Children's Hospital, 705 Riley Hospital Drive, ROC 4270, Indianapolis, IN-46202, USA, Tel: (317) 948-7769; Fax: (317) 944-7247; E-mail: sddavis3@iu.edu

Received January 23, 2016; Accepted February 22, 2016; Published February 25, 2016

Citation: Assaf SJ, Clem CC, Jewett LB, Schornick L, Tiller CJ, et al. (2016) Ventilation Heterogeneity and Airway Hyperreactivity in Children with Well Controlled Asthma. J Pulm Respir Med 6: 321. doi:10.4172/2161-105X.1000321

Copyright: ( 2016 Assaf SJ, et al. This is an open-access article distributed under the terms of the Creative Commons Attribution License, which permits unrestricted use, distribution, and reproduction in any medium, provided the original author and source are credited. 
autoimmune, neoplastic, and/or congenital heart disease. Subjects were clinically healthy at the time of testing, with no fever or acute respiratory symptoms for 4 weeks prior to testing.

The study was approved by the Institutional Review Board at Indiana University and informed parental consent as well as assent in children $\geq 7$ years old was obtained.

\section{Nitrogen multiple breath washout $\left(\mathrm{N}_{2} \mathrm{MBW}\right)$}

$\mathrm{N}_{2} \mathrm{MBW}$ was assessed using a standard method according to current consensus [15] with Ecomedics ${ }^{\otimes}$ Exhalyzer D open circuit equipment and Spiroware ${ }^{\oplus}$ software (3.1.6) for data acquisition, storage, and analysis. $\mathrm{N}_{2} \mathrm{MBW}$ was performed with the child in a seated upright position, breathing through a mouthpiece and wearing nose clips while watching a video. The wash-in phase utilized nitrogen $\left(\mathrm{N}_{2}\right)$ as the tracer gas (room air bias flow), while the wash-out phase used $100 \%$ oxygen. The number of lung volume turnovers required to reduce $\mathrm{N}_{2}$ gas concentration to $1 / 40^{\text {th }}$ of the starting value was expressed as the lung clearance index (LCI), which was calculated as the ratio between the cumulative expired volume (CEV) and the mean functional residual capacity (FRC) calculated from the washout phase. Reported values were the average of 2-3 technically acceptable trials with no leaks or coughing during the washout time. LCI and FRC measurement coefficient of variability $(\mathrm{CV})$ was $\leq 5 \%$ for two trials and $\leq 10 \%$ for three trials.

\section{Spirometry and methacholine challenge}

Spirometry was measured by standardized methodology with the child standing and wearing nose clips [16,17]. At least three technically acceptable maneuvers were required with the $\mathrm{CV}$ of $\mathrm{FEV}_{1}$ being $\leq 10 \%$. Spirometry measurements were expressed as percent predicted based upon Global Lung Initiative (GLI) equations [18].

Methacholine bronchial challenge was only performed in children between 7-10 years of age. The five breath dosimeter protocol was performed according to American Thoracic Society 1999 Guidelines [19]. Children were seated throughout the testing and increasing methacholine concentrations $(0.0625,0.25,1,4$ and $16 \mathrm{mg})$ were nebulized and inhaled through a mouthpiece while wearing nose clips. The methacholine challenge was considered positive (airway hyper-reactivity present) when $\mathrm{FEV}_{1}$ decreased $\geq 20 \%$ from baseline and $\mathrm{PC}_{20}$, the methacholine concentration required to decrease $\mathrm{FEV}_{1}$ by $20 \%$, was calculated.

\section{Statistical analysis}

Subjects' demographics and lung function variables of each group (healthy controls and children with asthma) were summarized and compared using two-sample t-tests for continuous variables (all had normal distribution) and Chi-Square tests for categorical variables. Relationships between lung function measurements (LCI, FEV, $\mathrm{FEF}_{25}$. ${ }_{75}$ ) and the following covariates were evaluated using multiple regression models: age and subjects' group. Relationship between LCI and $\mathrm{PC}_{20}$ was evaluated using a linear regression model. For all the analyses, the level for statistical significance was set at 0.05 . All analytic assumptions were verified and all tests were performed using SPSS Statistics $22^{\circ}$ (SPSS, Chicago, IL, USA).

\section{Results}

\section{Demographics}

Forty-five children who met inclusion/exclusion criteria were evaluated; 24 were asthmatics and 21 were healthy. Table 1 summarizes the demographics for each group of subjects. There were no significant differences between the asthmatics and healthy groups in age, body length, weight, gender, or race.

\section{Lung function tests}

LCI was significantly higher (reflecting increased ventilation heterogeneity) in asthmatics compared to the healthy control subjects ( $\mathrm{p}=$ 0.004 , Table 2 ). In contrast, there were no statistically significant differences between the 2 groups for $\mathrm{FEV}_{1}(\mathrm{p}=0.16)$ and $\mathrm{FEF}_{25-75}(\mathrm{p}=0.19)$, although the asthmatic group tended to have lower spirometry values.

As a previous study had reported that LCI varied with age, we compared LCI versus age for the asthmatic and control subjects [20,21]. Neither asthmatics nor controls demonstrated a significant relationship between LCI and age; however, there was a significant difference in the slopes of LCI with age between the two groups $(\mathrm{p}=0.001)$ (Figure 1). For $\mathrm{FEV}_{1}$ and $\mathrm{FEF}_{25-75}$, neither group demonstrated significant relationships with age for either parameter, nor was there a difference between the slopes for the two groups.

\section{Airway hyperreactivity}

Twenty-seven children between 7 and 10 years of age ( 16 asthmatics and 11 healthy controls) were evaluated with both measurements of $\mathrm{N}_{2} \mathrm{MBW}$ and methacholine challenge; there were no demographic differences between asthmatics and the healthy controls in these groups that underwent methacholine challenge (Table 3). The group of asthmatic children had significantly higher LCI compared with healthy controls ( $p=0.008$ (Table 4), and tended to have lower FEV (percent predicted), which did not achieve statistical significance.

Nine asthmatics (56\%) and four healthy controls (36\%) had a positive response to methacholine $\left(\mathrm{PC}_{20}<16 \mathrm{mg} / \mathrm{ml}\right)$; however, there was no difference in the frequency of a positive test for the two groups $(\mathrm{p}$ $=0.31$ ). There was also not a significant difference in group mean $\mathrm{PC}_{20}$ for asthmatics compared to healthy control subjects (Table 4). Among the asthmatic subjects that had a positive methacholine challenge, there was a significant relationship between LCI and $\mathrm{PC}_{20}(\mathrm{p}=0.02)$;

\begin{tabular}{|c|c|c|c|}
\hline & Healthy Controls & Children with asthma & p-value \\
\hline Subjects (N) & 21 & 24 & \\
\hline Age (years) & $7.4(2.0)$ & $7.7(1.7)$ & 0.54 \\
\hline Height (cm) & $123(12.5)$ & $126(9.9)$ & 0.46 \\
\hline Weight (kg) & $26(6.88)$ & $29(9.74)$ & 0.25 \\
\hline $\begin{array}{c}\text { Gender(Female, } \\
\%)\end{array}$ & $5(24)$ & $7(29)$ & 0.68 \\
\hline $\begin{array}{c}\text { Race(Caucasian, } \\
\%)\end{array}$ & $18(86)$ & $21(87)$ & 0.86 \\
\hline $\begin{array}{l}\text { Values are means (standard deviations) for continuous variables; frequency } \\
\text { (percent) for categorical variables. P-values were derived from t-tests for } \\
\text { continuous variables, Chi-Square tests for categorical variables. }\end{array}$ \\
\hline
\end{tabular}

Table 1: Demographics.

\begin{tabular}{|c|c|c|c|}
\hline & Healthy Controls & $\begin{array}{c}\text { Children with } \\
\text { asthma }\end{array}$ & p-value \\
\hline Subjects $(\mathrm{N})$ & 21 & 24 & \\
\hline LCl & $6.79(0.59)$ & $7.58(1.03)$ & 0.004 \\
\hline FEV $_{1}(\%$ predicted $)$ & $107(10.29)$ & $102(13.22)$ & 0.16 \\
\hline FEF $_{25-75}(\%$ predicted $)$ & $97(19.59)$ & $85(25.22)$ & 0.19 \\
\hline
\end{tabular}

Values are means (standard deviations) for continuous variables. P-values are derived from t-tests for continuous variables. 


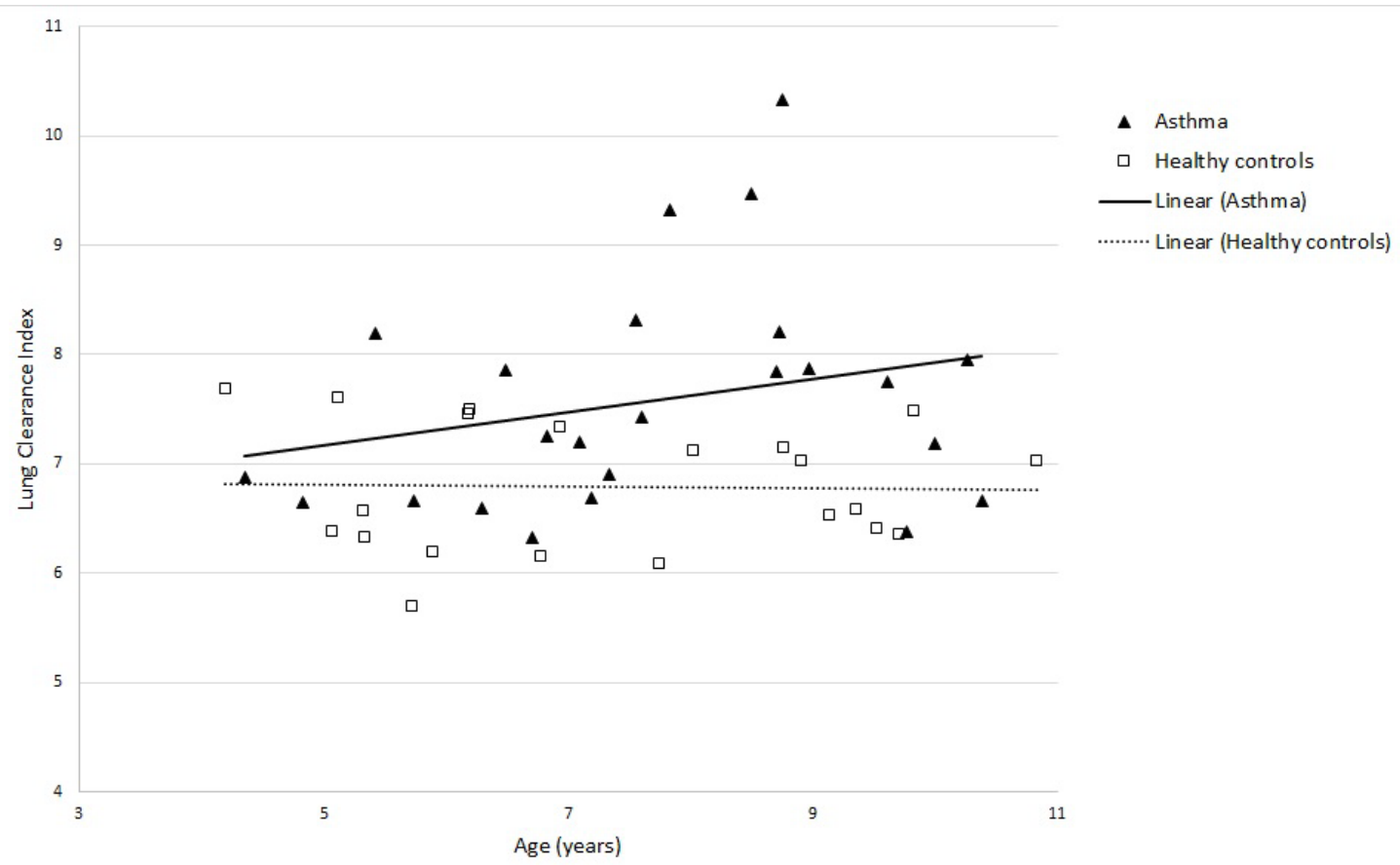

Figure 1: Lung Clearance Index (LCl) versus Age (years). Individual data for subjects with asthma (solid triangles) and control subjects (open squares) are presented, as well as the linear regressions for each group. The slopes of $\mathrm{LCl}$ versus age were significantly different between the asthmatics and healthy control subjects $(p=0.001)$, although there were not significant correlations between LCl and Age for asthmatics $(p=0.24)$ or healthy control $(p=0.92)$ groups.

\begin{tabular}{|c|c|c|c|}
\hline & Healthy Controls & $\begin{array}{c}\text { Children with } \\
\text { asthma }\end{array}$ & p-value \\
\hline Subjects (N) & 11 & 16 & \\
\hline Age (years) & $8.98(1.09)$ & $8.64(1.13)$ & 0.44 \\
\hline Height (cm) & $133(8.68)$ & $130(8.54)$ & 0.43 \\
\hline Weight (kg) & $32(6.87)$ & $32(10.15)$ & 0.91 \\
\hline Gender (Female, \%) & $2(18)$ & $4(25)$ & 0.67 \\
\hline Race (Caucasian, \%) & $10(91)$ & $14(87)$ & 0.78 \\
\hline
\end{tabular}

Values are means (standard deviations); P-values are derived from t-tests. * Values are number, $\mathrm{N}$, and frequency (percent); P-value are derived from Chisquare tests.

Table 3: Methacholine challenge group (demographics).

\begin{tabular}{|c|c|c|c|}
\hline & Healthy Controls & $\begin{array}{c}\text { Children with } \\
\text { asthma }\end{array}$ & p-value \\
\hline Subjects $(\mathrm{N})$ & 11 & 16 & \\
\hline $\mathrm{LCl}$ & $6.83(0.45)$ & $7.41(0.63)$ & 0.008 \\
\hline Baseline $\mathrm{FEV}_{1}(\%$ predicted $)$ & $106(9.45)$ & $100(9.81)$ & 0.08 \\
\hline Baseline $\mathrm{FEF}_{25-75}(\%$ predicted) & $93(19.53)$ & $86(22.34)$ & 0.18 \\
\hline $\mathrm{PC}_{20}$ reactive $(\mathrm{N} ; \%)$ & $4(36 \%)$ & $9(56 \%)$ & 0.31 \\
\hline $\mathrm{PC}_{20}$ reactive $(\mathrm{mg} / \mathrm{ml})$ & $5.31(4.87)$ & $5.79(4.72)$ & 0.87 \\
\hline $\mathrm{PC}_{20}$ reactive $(\mathrm{FEV} \%$ drop$)$ & $34.5(25.05)$ & $32.3(7.96)$ & 0.67 \\
\hline
\end{tabular}

Values are means (standard deviations); P-values are derived from t-tests.

*Values are number, $\mathrm{N}$, and frequency (percent) with positive bronchial challenge; P-value are derived from Chi-square tests.

Table 4: Methacholine challenge. increasing LCI (greater ventilation heterogeneity) was associated with a lower $\mathrm{PC}_{20}$ (greater airway reactivity) (Figure 2). When we analyzed all asthmatic subjects, responders and non-responders $(n=16)$, the relationship between increasing $\mathrm{LCI}$ and lower $\mathrm{PC}_{20}$ approached statistical significance $(p=0.07)$. Among healthy control subjects there was no significant relationship between LCI and $\mathrm{PC}_{20}$ (Figure 2). Neither asthmatics nor healthy controls subjects had a significant relationship between $\mathrm{FEF}_{25-75}$ or $\mathrm{FEV}_{1}$ (percent predicted) and $\mathrm{PC}_{20}$.

\section{Discussion}

Ventilation heterogeneity within the lung is a well-recognized feature of asthma; however, relatively few studies have evaluated this measure in young asthmatic children $[10,22,23]$. In our study, we found that not only did young well-controlled children with asthma have higher LCI values when compared with healthy controls, but there was also a significant relationship between LCI and airway reactivity among the asthmatic children; increasing LCI was associated with greater airway reactivity.

Our well-controlled children with asthma had significantly higher LCI values compared to healthy controls, reflecting greater ventilation heterogeneity; however, differences in spirometry did not achieve statistical significance. These findings reinforce that LCI is a more sensitive measure of airway dysfunction than spirometry, particularly in children with well controlled disease. Our finding in pre-school and school age children is consistent with other studies of older children and adults with well controlled asthma [9]. However, this study is one of the first using nitrogen $\left(\mathrm{N}_{2}\right)$ as an inert gas in this age group, while previous studies [9-12] used different gases such as sulfur hexafluoride $\left(\mathrm{SF}_{6}\right)$. Given that $\mathrm{SF}_{6}$ is difficult to obtain in the United States, a better understanding of the sensitivity of measurements of MBW using $\mathrm{N}_{2}$ as the inert gas is important. We also found there was a difference in the relationship between LCI and age among our well-controlled pre-school and school age asthmatic children compared to healthy control subjects; the asthmatics had a greater increase in slope of LCI compared to the controls. Although our study 


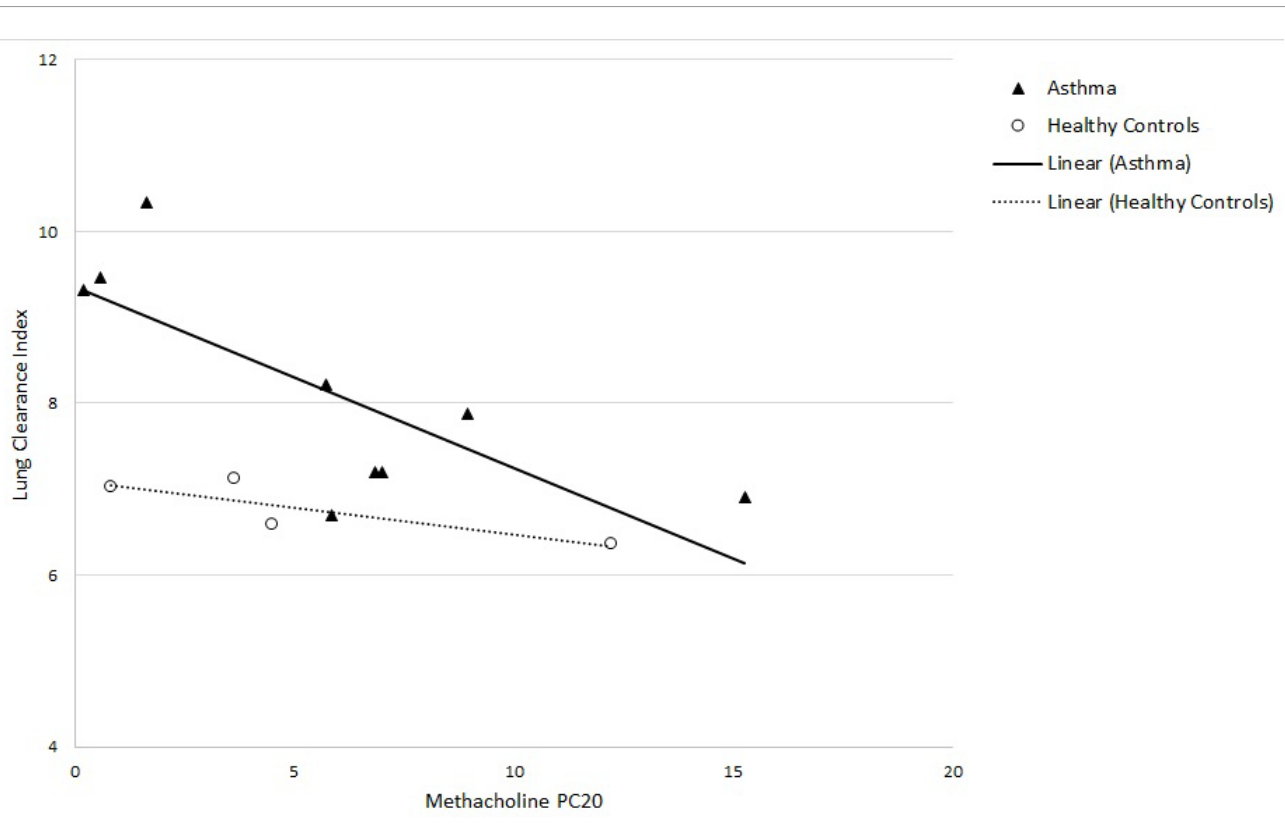

Figure 2: Lung Clearance Index ( $\mathrm{LCl}$ ) versus Airway Reactivity - $\mathrm{PC}_{20}$ ( $\left.\mathrm{mg} / \mathrm{ml}\right)$. Individual data for subjects with asthma (solid triangles) and control subjects (open circles) who were reactive to methacholine are presented, as well as the linear regressions for each group. The relationship between $\mathrm{LCl}_{\text {and }}$ methacholine $\mathrm{PC} \mathrm{C}_{20}$ was significant in asthmatics $(p=0.02)$; LCl increased with decreasing $\mathrm{PC}_{20}$ in asthmatic subjects; however, there was no significant relationship between $\mathrm{LCl}$ and $\mathrm{PC}{ }_{20}$ in healthy controls $(p=0.16)$.

was cross-sectional, rather than longitudinal, our findings suggest that even among well-controlled asthmatic children, ventilation heterogeneity may increase with increasing age. Similar findings were observed with spirometric values for which longitudinal cohort studies suggest worsening airway obstruction with increasing age [24-26]. Our findings suggest that LCI may be more sensitive than spirometry for detecting worsening airway function in well-controlled asthmatic children. Earlier detection of disease prior to airway remodeling may help to alter the trajectory of disease.

As a group, our children with asthma did not have significantly greater airway reactivity compared to our healthy control subjects, which may reflect selection bias since 15 of the 16 that underwent bronchial challenge were using inhaled corticosteroids that can suppress airway reactivity. We did find that among the asthmatic children, but not the healthy controls, increasing ventilation heterogeneity was associated with greater airway reactivity (lower $\mathrm{PC}_{20}$ ). Our findings are in agreement with previous studies in adults that demonstrated similar relationships between ventilation heterogeneity and airway reactivity [8]; however, our study has extended this finding from adults to young children, particularly, those with well-controlled asthma. In adults with clinically unstable asthma, Downie et al. demonstrated the persistence of this relationship following anti-inflammatory treatment, which suggested that ventilation heterogeneity may be an important independent determinant of airway hyperresponsiveness. Computational models of airway narrowing have also suggested that inhomogeneity of airway obstruction, which contributes to ventilation heterogeneity, might be associated with increased airway reactivity [2729]. Therefore, normalization of ventilation heterogeneity may be an important treatment goal for subjects with asthma. However, at what age ventilation heterogeneity develops, and whether it precedes airway hyper-reactivity or develops concurrently is not known.

Our study has several limitations. The sample size was low for those that performed methacholine challenge, especially amongst the healthy controls. We did not measure ventilation heterogeneity at the end of the methacholine challenge; we only measured spirometry. Given this, we were unable to determine whether a change in LCI was associated with the change in spirometry. We administered the bronchodilator immediately after the last methacholine dose and spirometry maneuver so as to not maintain the airway obstruction in these young subjects, who were also not likely to tolerate a much longer protocol. Another limitation is that our study was cross-sectional; therefore, we were not able to determine whether LCI progressively worsens over time in well-controlled asthmatic children relative to healthy controls. Lastly, a longitudinal cohort study starting during infancy is required to determine whether ventilation heterogeneity precedes or develops coincidentally with heightened airway reactivity.

In conclusion, we found that ventilation heterogeneity measured using $\mathrm{N}_{2}$ washout, unlike spirometry, demonstrated airway dysfunction in young well-controlled children with asthma. Further, increasing ventilation heterogeneity was related to increasing airway reactivity in young wellcontrolled children with asthma, but not in healthy controls. Future studies are required to determine the mechanistic relationship between ventilation heterogeneity and airway reactivity in children with asthma, as well as whether ventilation heterogeneity can be an important outcome for assessing the management of children with asthma.

\section{Acknowledgements}

The authors would like to thank all study participants and their families. The authors have no conflict of interest to declare.

\section{Grants}

Cystic Fibrosis Foundation Therapeutics, Inc. Core Lab grant DAVIS08. Indiana Physician Scientist Award, Eli Lilly grant.

\section{References}

1. Rubin BK, Priftis KN, Schmidt HJ, Henke MO (2014) Secretory hyperresponsiveness and pulmonary mucus hypersecretion. Chest 146: 496507.

2. Regamey N, Jeffery PK, Alton EW, Bush A, Davies JC (2011) Airway remodelling 
Citation: Assaf SJ, Clem CC, Jewett LB, Schornick L, Tiller CJ, et al. (2016) Ventilation Heterogeneity and Airway Hyperreactivity in Children with Well Controlled Asthma. J Pulm Respir Med 6: 321. doi:10.4172/2161-105X.1000321

Page 5 of 5

and its relationship to inflammation in cystic fibrosis. Thorax 66: 624-629.

3. Macklem PT (1998) The physiology of small airways. Am J Respir Crit Care Med 157: S181-183.

4. Stocks J, Thia LP, Sonnappa S (2012) Evaluation and use of childhood lung function tests in cystic fibrosis. Curr Opin Pulm Med 18: 602-608.

5. Fuchs SI, Gappa M (2011) Lung clearance index: clinical and research applications in children. Paediatr Respir Rev 12: 264-270.

6. Verbanck S, Schuermans D, Paiva M, Vincken W (2003) Nonreversible conductive airway ventilation heterogeneity in mild asthma. J Appl Physiol (1985) 94: 1380-1386.

7. Verbanck S, Paiva M, Schuermans D, Hanon S, Vincken W, et al. (2012) Relationships between the lung clearance index and conductive and acinar ventilation heterogeneity. J Appl Physiol (1985) 112: 782-790.

8. Downie SR, Salome CM, Verbanck S, Thompson B, Berend N, et al. (2007) Ventilation heterogeneity is a major determinant of airway hyperresponsiveness in asthma, independent of airway inflammation. Thorax 62: 684-689.

9. Zwitserloot A, Fuchs SI, Müller C, Bisdorf K, Gappa M (2014) Clinical application of inert gas Multiple Breath Washout in children and adolescents with asthma. Respir Med 108: 1254-1259.

10. Macleod KA, Horsley AR, Bell NJ, Greening AP, Innes JA, et al. (2009) Ventilation heterogeneity in children with well controlled asthma with normal spirometry indicates residual airways disease. Thorax 64: 33-37.

11. Robinson PD, Stocks J, Aurora P, Lum S (2013) Abbreviated multi-breath washout for calculation of lung clearance index. Pediatr Pulmonol 48: 336-343.

12. Sigurs N, Aljassim F, Kjellman B, Robinson PD, Sigurbergsson F, et al. (2010) Asthma and allergy patterns over 18 years after severe RSV bronchiolitis in the first year of life. Thorax 65: 1045-1052.

13. www.ginasthma.org.

14. Liu AH, Zeiger R, Sorkness C, Mahr T, Ostrom N, et al. (2007) Development and cross-sectional validation of the Childhood Asthma Control Test. J Allergy Clin Immunol 119: 817-825.

15. Robinson PD, Latzin P, Verbanck S, Hall GL, Horsley A, et al. (2013) Consensus statement for inert gas washout measurement using multiple- and singlebreath tests. Eur Respir J 41: 507-522.

16. Beydon N, Davis SD, Lombardi E, Allen JL, Arets HG, et al. (2007) An official American Thoracic Society/European Respiratory Society statement: pulmonary function testing in preschool children. Am J Respir Crit Care Med 175: 1304-1345.

17. Miller MR, Hankinson J, Brusasco V, Burgos F, Casaburi R, et al. (2005) ATS/ ERS Task Force: Standardisation of lung function testing. Standardisation of spirometry. Eur Respir J 26: 319-338.

18. Quanjer PH, Stanojevic S, Cole TJ, Baur X, Hall GL, et al. (2012) Multi-ethnic reference values for spirometry for the 3-95-yr age range: the global lung function 2012 equations. Eur Respir J 40: 1324-1343.

19. Crapo RO, Casaburi R, Coates AL, Enright PL, Hankinson JL, et al. (2000) Guidelines for methacholine and exercise challenge testing-1999. This official statement of the American Thoracic Society was adopted by the ATS Board of Directors, July 1999. Am J Respir Crit Care Med 161: 309-329.

20. Chakr VC, Llapur CJ, Sarria EE, Mattiello R, Kisling J, et al. (2012) Ventilation homogeneity improves with growth early in life. Pediatr Pulmonol 47: 373-380.

21. Lum S, Stocks J, Stanojevic S, Wade A, Robinson P, et al. (2013) Age and height dependence of lung clearance index and functional residual capacity. Eur Respir J 41: 1371-1377.

22. Gustafsson PM (2007) Peripheral Airway Involvement in CF and Asthma Compared by Inert Gas Washout. Ped Pulmonol 42: 168-176.

23. Sonnappa S, Bastardo CM, Wade A, Bush A, Stocks J, et al. (2013) Repeatability and bronchodilator reversibility of lung function in young children. Eur Respir J 42: 116-124.

24. Brown PJ, Greville HW, Finucane KE (1984) Asthma and irreversible airflow obstruction. Thorax 39: 131-136.

25. Morgan WJ, Stern DA, Sherrill DL, Guerra S, Holberg CJ, et al. (2005) Outcome of asthma and wheezing in the first 6 years of life: follow-up through adolescence. Am J Respir Crit Care Med 172: 1253-1258.

26. Bisgaard H, Jensen SM, Bønnelykke K (2012) Interaction between asthma and lung function growth in early life. Am J Respir Crit Care Med 185: 1183-1189.

27. Venegas JG, Winkler T, Musch G, Vidal Melo MF, Layfield D, et al. (2005) Selforganized patchiness in asthma as a prelude to catastrophic shifts. Nature 434 777-782.

28. Thorpe CW, Bates JH (1997) Effect of stochastic heterogeneity on lung impedance during acute bronchoconstriction: a model analysis. J Appl Physiol (1985) 82: 1616-1625.

29. Lutchen KR, Gillis H (1997) Relationship between heterogeneous changes in airway morphometry and lung resistance and elastance. J Appl Physiol (1985) 83: 1192-1201. 